\title{
Brechas socioeconómicas, generacionales y de género en la educación peruana: una aproximación con datos masivos de una ciudad norteña
}

\section{Gaps in socio-economic, generational and gender in the Peruvian education: An approach with mass data of a northern city}

\author{
Wendy Arhuis-Inca ${ }^{1 a}$, Juan Carlos Bazo-Alvarez², Janina Bazalar-Palacios ${ }^{3}$, \\ Miguel Ipanaqué-Zapata ${ }^{4}$ \\ Universidad Católica Los Ángeles de Chimbote, Perú ${ }^{1,2,3,4}$ \\ Instituto de Investigación, Capacitación y Desarrollo Psicosocial y Educativo \\ (PSYCOPERU), Lima, Perú \\ (iD) ORCID ID: https://orcid.org/0000-0002-5317-5053 ${ }^{1}$ \\ (iD) ORCID ID: https://orcid.org/0000-0002-6169-8049² \\ iD ORCID ID: https://orcid.org/0000-0001-8515-0742 3 \\ ORCID ID: https://orcid.org/0000-0001-8706-6018
}

Recibido: 14 de enero de 2021

Aceptado: 28 de marzo de 2021

\begin{abstract}
Resumen
El objetivo de esta investigación fue determinar la brecha educativa entre géneros, niveles socioeconómicos y grupos generacionales en adultos citadinos. El diseño fue observacional y transversal, utilizando datos del Estudio Poblacional 2016-2017 realizado en Chimbote, Perú. Se observó el logro educativo, sexo, nivel socioeconómico y edad (grupo generacional) de 20,005 personas mayores de 22 años. Los datos fueron resumidos en tablas de contingencia y analizados con la Chi-cuadrado de homogeneidad. Los resultados determinaron que las personas adultas presentaron brecha educativa, evidenciando claro desfavorecimiento en el nivel socioeconómico bajo, notándose en los grupos de nivel educativo secundario y superior. Dentro de los niveles socioeconómicos existen desigualdades educativas, evidenciándose en el logro secundario y superior para las mujeres más pobres y en el nivel superior para las generaciones menos jóvenes de ambos niveles socioeconómicos.
\end{abstract}

Palabras clave: Brecha; género; nivel socioeconómico; nivel educativo; adulto.

\begin{abstract}
Our aim was to determine the educational gap according to gender, socioeconomic status, and generational group among city adults. The study design was observational and cross-sectional, using data from the 2016-2017 Population Study in Chimbote, Peru. We looked at educational
\end{abstract}


attainment, sex, socioeconomic status and age (for defining generational groups) of 20,005 people aged 22 years and over. Data were summarized in cross-tables and analyzed with a Chisquare test of homogeneity. The results showed that adults presented an educational gap, with a clear disadvantage in the lower socioeconomic level, particularly in the secondary and higher education level groups. Within the socioeconomic levels there are educational inequalities, evidenced in secondary and higher achievement for the poorest women and at the higher level for the younger generations of both socioeconomic levels.

Keywords: Gap; gender; socioeconomic level; education level; adult.

\section{Introducción}

Las brechas de género refieren a las desigualdades en derechos, responsabilidades y oportunidades entre hombres y mujeres, y tales brechas vienen siendo reducidas en el Perú y el mundo (United Nations Educational Scientific and Cultural Organization (UNESCO), 2019). Entre otras cosas, estas desigualdades inciden en el acceso a la educación (tener la oportunidad de estudiar) y el logro educativo (qué tanto se avanzó en los estudios), afectando principalmente a las mujeres (Instituto Nacional de Estadística e Informática del Perú (INEI), 2020). En el 2016 se evaluó a 144 países, de los cuales el 95\% redujeron la brecha en educación entre hombres y mujeres respecto a años anteriores (World Economic Forum, 2016). El mismo año, el gobierno peruano invirtió en educación 10.1\% más que el año anterior para reducir la brecha educativa (INEI, 2017). De hecho, una mayor equidad entre géneros ya es evidente en el acceso a la educación inicial y primaria (INEI, 2017; World Economic Forum, 2016). Sin embargo, la brecha sigue favoreciendo a los hombres en la educación secundaria y superior (en universidades nacionales y en ciertas carreras) (INEI, 2017). A pesar que los hombres siguen estando favorecidos, las mujeres tienen mayor participación que hace una década (Benavides, 2007; INEI, 2017; Comisión Económica para América Latina y el Caribe (CEPAL), 2017; UNESCO, 2020).

Por otro lado, las desigualdades socioeconómicas también afectan el acceso a la educación y el logro educativo (Akabayashi, et al., 2020; Lundberg, 2020; Vaezghasemi, et al., 2020). Decomposition of income-related inequality in upper secondary school completion in Sweden by mental health, family conditions and contextual characteristics. SSM-population health, 11, 100566.). En el 2017 se reportó un acceso equitativo en el nivel de primaria, pero en el nivel de secundaria las brechas aún fueron patentes: el quintil más pobre presentó 15,1\% menos acceso que el quintil más rico (INEI, 2018). El nivel socioeconómico también condiciona las brechas de género, aunque de formas distintas entre primaria y secundaria (Bodovski, et al., 2020). Para el nivel primario, los hombres lideran en el quintil más pobre, 
mientras que las mujeres lideran en el quintil más rico (INEI, 2017). Para el nivel secundario, los hombres siguen favorecidos en ambos quintiles socioeconómicos (INEI, 2017). Respecto a la educación superior, los datos actuales sobre las brechas por condición socioeconómica son escasos (Díaz, 2008; Guadalupe, et al., 2017; INEI, 2017; INEI, 2018).

En este contexto, la brecha educativa también existe entre grupos generacionales, donde idealmente las nuevas generaciones deberían tener mayor acceso y logro educativo (Angrisani, et al., 2020). En el 2016, los estudiantes matriculados entre 6-8 años alcanzaron un poco más del 90\%, mientras que para el periodo 1993-2005 no superaban el 70\% (Guadalupe et al., 2017). Esto implica que la generación más joven (v.g. 2016) tuvo más acceso que sus contrapartes más antiguas (v.g.1993), por lo que se esperaría que la más joven logre alcanzar mayores niveles educativos. De hecho, los estudiantes matriculados en secundaria de colegios estatales (12-16 años) en el 2016 superaron por 84\% a los del periodo 1993 (Guadalupe et al., 2017). No obstante, el factor socioeconómico también puede afectar estos avances. Por ejemplo, a pesar que entre el 2009 y 2019 el analfabetismo se redujo en 2,0\%, la mayor proporción de personas que no saben leer y escribir siguen estando en zonas rurales (INEI, 2020). El problema de cómo las diferencias socioeconómicas afectan las ventajas que las nuevas generaciones deben tener en cuanto al acceso y logro educativo aún no ha sido explorado de forma suficiente.

Por todo lo mencionado, el objetivo del presente estudio ha sido determinar la brecha educativa según género, nivel socioeconómico y grupo generacional, en pobladores adultos de la ciudad de Chimbote-Perú, a partir de datos masivos provenientes de un estudio poblacional realizado en dicha ciudad.

\section{Metodología}

\section{Diseño y contexto del estudio}

Estudio observacional y transversal, desarrollado como un análisis secundario de la base de datos del Estudio Poblacional de Chimbote 2016-2017 (Domínguez, et al., 2019). Dicho estudio fue diseñado originalmente para determinar la prevalencia por autoreporte de enfermedades crónicas en Chimbote-Perú, y caracterizar el perfil bio-sociodemográfico de sus habitantes. No obstante, el estudio legó una gran cantidad de data que permite explorar información relevante sobre distintos temas: 63731 encuestados de una población estimada de 135455 (INEI, 2018). 


\section{Procedimientos}

El estudio primario recolectó la información durante el periodo diciembre 2015-enero 2017. Para ello, se capacitó previamente al personal encargado de la aplicación de las encuestas, quienes tuvieron como funciones: i) identificar correctamente la casa seleccionada (de no encontrar algún miembro del hogar, debían retornar como máximo 2 veces); ii) identificar al jefe del hogar (miembro mayor de 18 años quien reportó tener la responsabilidad de la administración del hogar); iii) informarle acerca del estudio a través del consentimiento informado, con el fin de verificar su aceptación; iv) aplicar la encuesta. Un equipo de supervisión realizó la gestión de los datos en el campo (para maximizar la recolección) y en oficina (para el registro).

\section{Participantes}

El estudio poblacional de Chimbote incluyó una muestra probabilística de 63,731 encuestados, cuyo procedimiento de selección se detalla en el estudio original (Domínguez et al., 2019). Para el presente estudio, se retiraron las siguientes observaciones: 210 duplicadas, 1111 inconsistentes, 117 sin registro de nivel educativo, 27753 sin registro del proxy socioeconómico y 14535 que eran menores de 22 años. Este último criterio de exclusión por edad obedece a que nuestro estudio se enfoca en las brechas educativas que afectan hoy a quienes son adultos, diferenciándose así de los reportes que el Ministerio de Educación emite regularmente. De esa forma, los participantes que observamos en nuestro estudio hicieron un total de 20005 adultos residentes de la ciudad de Chimbote. Esta ciudad-puerto, ubicada en la costa norte del Perú, posee características étnicas y culturales muy similares a otras ciudades de esa región. La mayoría de sus habitantes son hispano hablantes y se desempeñan en actividades ligadas a la industria pesquera, la metalúrgica, la agricultura o el comercio.

\section{Variables}

La variable dependiente nivel educativo se definió como "el grado alcanzado por una persona en el sistema educativo formal” (Romero-Martínez, Montoro-Sánchez Y GaravitoHernández, 2017). Esta variable se categorizó como: sin estudios, nivel primario, nivel secundario, y nivel superior. Las variables independientes fueron: sexo (masculino y femenino); edad (recolectada como fecha de nacimiento y luego categorizada en 22-32 años, 32-42 años, 42-52 años, 52-62 años y $\geq 62$ años); nivel socioeconómico (alto y bajo), que no fue medida sino aproximada a partir de la variable tenencia de celular inteligente (Steele et al., 2018). Esta 
última proviene de la pregunta: ¿'Tiene un smartphone o celular inteligente? con respuesta en 2 categorías (Si/No).

\section{Plan de análisis}

Se realizó un análisis bivariado mediante tablas de contingencia, donde se cruzaron las variables nivel socioeconómico (SES) y nivel educativo (Tabla 1). Se adicionó la variable sexo a esta tabla para estratificar a los grupos ya estratificados según SES, construyendo así una nueva tabla de contingencia (Tabla 2). Luego, se hizo lo propio, pero estratificando por edad en vez de sexo (Tabla 3). T odas las tablas se reportaron porcentajes por filas, específicamente se mostró según niveles SES para la Tabla 1 y al interior de cada nivel SES según sexo y edad para las Tablas 2 y 3 respectivamente, adicionalmente se calculó la prueba Chi cuadrado de homogeneidad debido que se tiene como objetivo reportar si las subpoblaciones dentro del SES muestran distribuciones similares según nivel educativo, siendo significativo con $\mathrm{p}<0.01$. El procesamiento y análisis de los datos se realizó con ayuda del software estadístico Stata 15.0 (StataCorp, 2017).

\section{Consideraciones éticas}

Desde que el presente es un estudio con análisis secundario de datos, no se tuvo contacto alguno con los participantes; por lo tanto, los posibles riesgos para ellos fueron mínimos. No obstante, se respetó la confidencialidad ofrecida en el consentimiento del estudio original, a través de la anonimización de los datos, su manejo restringido y registro encriptado. Ambos estudios, el original y el presente, fueron revisados por el Comité Institucional de Ética en Investigación de la Universidad Católica Los Ángeles de Chimbote (CIEI-ULADECHCatólica), quien emitió sendos informes de autorización: º006-2016-CEI-VI-ULADECHCatólica (estudio original) y N017-2018-CIEI-VI-ULADECH-Católica (presente estudio).

\section{Resultados}

Se observó un total de 20005 personas mayores de 22 años, 49,9\% mujeres, 79,0\% con SES alto y $21,0 \%$ con SES bajo. 


\section{Tabla 1}

Nivel educativo según nivel socioeconómico

\begin{tabular}{|c|c|c|c|c|c|c|c|c|}
\hline \multirow[b]{2}{*}{ SES } & \multicolumn{5}{|c|}{ Nivel educativo } & \multirow[b]{2}{*}{ n (\%) } & \multirow[b]{2}{*}{$\mathrm{X}^{2}$} & \multirow[b]{2}{*}{$\mathbf{P}$} \\
\hline & $\begin{array}{c}\text { Sin } \\
\text { Estudios } \\
(\%)\end{array}$ & $\begin{array}{c}\text { Nivel } \\
\text { Inicial } \\
(\%)\end{array}$ & $\begin{array}{c}\text { Nivel } \\
\text { Primario } \\
(\%)\end{array}$ & $\begin{array}{c}\text { Nivel } \\
\text { Secundario } \\
\text { (\%) }\end{array}$ & $\begin{array}{c}\text { Nivel } \\
\text { Superior } \\
(\%)\end{array}$ & & & \\
\hline Alto & 0,44 & 0,04 & 9,64 & 47,86 & 42,02 & $15,805(100,0)$ & & \\
\hline Bajo & 9,33 & 0,36 & 32,24 & 38,69 & 19,38 & $4,200(100,0)$ & 293,0 & $<0,001$ \\
\hline Total & 2,3 & 0,11 & 14,38 & 45,94 & 37,27 & $20,005(100,0)$ & & \\
\hline
\end{tabular}

Fuente: elaboración propia

En la tabla 1 se observó una mayor proporción de las personas con SES alto alcanzaron una educación de nivel secundario (SES-Alto=47,9\% versus SES-Bajo=38,7\%) y superior (SES-Alto=42,0\% versus SES-Bajo=19,4\%) (Tabla 1). Esta tendencia se invirtió sólo en los niveles educativos más bajos: primaria (SES-Alto=9,6\% versus SES-Bajo=32,2\%), inicial (SES-Alto=0,04\% versus SES-Bajo=0,36\%) y sin estudios (SES-Alto=0,44\% versus SESBajo=9,3\%). Asimismo, según el reporte Chi-cuadrado donde se tuvo como hipótesis nula si las distribuciones de los niveles educativos son similares según SES y se obtuvo que se rechaza dicha hipótesis reportando que si existe diferencias entre dichos grupos poblacionales según SES [X2[4]=293,0; $<<0,001]$.

\section{Tabla 2}

Nivel educativo según género y nivel socioeconómico

\section{Nivel educativo}

\begin{tabular}{cccccccccc} 
SES & Género & $\begin{array}{c}\text { Sin Nivel } \\
(\%)\end{array}$ & $\begin{array}{c}\text { Nivel } \\
\text { Inicial } \\
(\%)\end{array}$ & $\begin{array}{c}\text { Nivel } \\
\text { Primaria } \\
(\%)\end{array}$ & $\begin{array}{c}\text { Nivel } \\
\text { Secundaria } \\
(\%)\end{array}$ & $\begin{array}{c}\text { Nivel } \\
\text { Superior } \\
(\%)\end{array}$ & n (\%) & $\mathbf{X}^{\mathbf{2}}$ & P \\
\hline \multirow{3}{*}{ Alto } & Femenino & 0,57 & 0,08 & 10,06 & 47,66 & 41,64 & $7,745(100,0)$ & & \\
& Masculino & 0,31 & 0,01 & 9,23 & 48,06 & 42,38 & $8,060(100,0)$ & 13,3 & 0,01 \\
& Total & $\mathbf{0 , 4 4}$ & $\mathbf{0 , 0 4}$ & $\mathbf{9 , 6 4}$ & $\mathbf{4 7 , 8 6}$ & $\mathbf{4 2 , 0 2}$ & $\mathbf{1 5 , 8 0 5 ( 1 0 0 , 0 )}$ & & \\
\multirow{2}{*}{ Bajo } & Femenino & 12,58 & 0,36 & 33,27 & 35,55 & 18,24 & $2,242(100,0)$ & & \\
& Masculino & 5,62 & 0,36 & 31,05 & 42,29 & 20,68 & $1,958(100,0)$ & 71,3 & 0,001 \\
& Total & $\mathbf{9 , 3 3}$ & $\mathbf{0 , 3 6}$ & $\mathbf{3 2 . 2 4}$ & $\mathbf{3 8 , 6 9}$ & $\mathbf{1 9 , 3 8}$ & $\mathbf{4 , 2 0 0 ( \mathbf { 1 0 0 , 0 } )}$ & \\
\hline
\end{tabular}

Fuente: elaboración propia 
En la tabla 2, se evaluó dos hipótesis nulas (para cada SES) si las distribuciones de las categorías del sexo son similares según sus niveles educativos son similares. El nivel SES alto reportó que se aceptó la hipótesis nula interpretándose que las distribuciones poblacionales de hombres y mujeres son similares según los niveles educativos [X2[4]=13,3; $p=0,01]$. Mientras que en el grupo con SES bajo se rechazó la hipótesis nula mostrando tener brechas educativas según sexo, todo ello se evidenció notoriamente en los niveles de secundaria y superior teniendo una clara desventaja del logro educativo en las mujeres con respecto a los hombres $[\mathrm{X} 2[4]=71,3 ; \mathrm{p}<0,001]$. Por otro lado, las mujeres pertenecientes al SES alto mostraron mayor desarrollo educativo, comparadas con las mujeres con SES bajo (nivel secundario + nivel superior: SES-Alto= 89,3\% versus SES-Bajo=53,8\%).

\section{Tabla 3}

Nivel educativo según edad y nivel socioeconómico

\begin{tabular}{|c|c|c|c|c|c|c|c|c|c|}
\hline \multirow[b]{2}{*}{ SES } & \multirow[b]{2}{*}{ Edad } & \multicolumn{6}{|c|}{ Nivel Educativo } & \multirow[b]{2}{*}{$X^{2}$} & \multirow[b]{2}{*}{$\mathbf{P}$} \\
\hline & & $\begin{array}{l}\text { Sin } \\
\text { Nivel } \\
\text { (\%) }\end{array}$ & $\begin{array}{c}\text { Nivel } \\
\text { Inicial } \\
(\%)\end{array}$ & $\begin{array}{c}\text { Nivel } \\
\text { Primaria } \\
\text { (\%) }\end{array}$ & $\begin{array}{c}\text { Nivel } \\
\text { Secundaria } \\
\text { (\%) }\end{array}$ & $\begin{array}{c}\text { Nivel } \\
\text { Superior } \\
\text { (\%) }\end{array}$ & n (\%) & & \\
\hline \multirow{5}{*}{ Alto } & $22-32$ & 0,11 & 0,02 & 3,84 & 41,89 & 54,14 & $6,202(100,0)$ & \multirow{5}{*}{203,0} & \multirow{5}{*}{$<0.001$} \\
\hline & $32-42$ & 0,19 & 0,02 & 7,12 & 53,55 & 39,12 & $4,116(100,0)$ & & \\
\hline & $42-52$ & 0,51 & 0,1 & 10,74 & 54,64 & 34,01 & $3,111(100,0)$ & & \\
\hline & $52-62$ & 0,98 & 0 & 20,5 & 49,28 & 29,24 & $1,532(100,0)$ & & \\
\hline & $\geq 62$ & 2,73 & 0,24 & 40,76 & 36,49 & 19,79 & $844(100,0)$ & & \\
\hline \multirow{7}{*}{ Bajo } & Total & 044 & 0,04 & 9,64 & 47,86 & 42,02 & $15,805(100,0)$ & \multirow{7}{*}{140,3} & \multirow{7}{*}{$<0.001$} \\
\hline & $22-32$ & 2,26 & 0,13 & 8,18 & 47,3 & 42,14 & $795(100,0)$ & & \\
\hline & $32-42$ & 3,37 & 0,64 & 14,13 & 55,06 & 26,81 & $623(100,0)$ & & \\
\hline & $42-52$ & 4,86 & 0,74 & 22,53 & 51,99 & 19,88 & $679(100,0)$ & & \\
\hline & $52-62$ & 5,96 & 0 & 35,27 & 43,64 & 15,14 & $621(100,0)$ & & \\
\hline & $\geq 62$ & 19,1 & 0,34 & 55,94 & 19,03 & 5,6 & $1,482(100,0)$ & & \\
\hline & Total & 9,33 & $\mathbf{0}$ & 32 & 38,69 & 19,38 & $4,200(100,0)$ & & \\
\hline
\end{tabular}

Fuente: Elaboración propia

En la Tabla 3, también se evaluó una hipótesis nula según SES sobre si las distribuciones son similares para las categorías de grupos generacionales según nivel educativo. En ambos niveles SES, se rechazó las hipótesis nula reportando que existen diferencias de distribución entre los grupos generaciones según niveles educativos, evidenciándose notoriamente que los más jóvenes tienen un logran alcanzar un mayor desarrollo educativo en comparación a los otros grupos de generacionales ( SES Alto:X2[16]=203,0; p<0,001 y SES Bajo: X2[16] =140,3; p<0,001). En particular, nótese el nivel superior donde los más jóvenes (22 a 32 años) tienen 
un mayor porcentaje (SES-Alto: 54,14\% y SES-Bajo: 42,14\%) que cualquiera de los grupos con más edad, especialmente el de mayores $\geq 62$ años (SES-Alto: 19,79\% y SES-Bajo: 5,6\%).

\section{Discusión}

El presente estudio exploró la brecha de educación según género, nivel socioeconómico y grupo generacional en pobladores adultos de la ciudad de Chimbote, Perú. Los adultos con SES alto mostraron un mayor nivel de logro educativo (secundaria y superior) que los adultos con SES bajo. En el grupo con SES bajo, las mujeres tuvieron menor nivel educativo que los hombres, particularmente visible en los niveles secundario y superior. Las mujeres con SES alto mostraron mayor desarrollo educativo que las mujeres con SES bajo. Las generaciones más jóvenes están logrando un mayor desarrollo educativo, con ventajas para aquellos con un SES alto.

\section{Brechas socioeconómicas en la educación}

La brecha socioeconómica en la educación entre adultos sigue existiendo, favoreciendo a quienes tienen más recursos económicos. Nuestros resultados destacan estas diferencias especialmente en el logro educativo a nivel secundario y superior. Estos resultados son consistentes con un estudio anterior de la OCDE (Organización para la Cooperación y el Desarrollo Económico), el cual reportó que, entre las personas de nivel socioeconómico alto, un 90\% tenía educación secundaria y un 50\% educación superior, en contraste con el 65\% y 10\% de las personas con nivel socioeconómico bajo (OCDE, 2016). Asimismo, éstos y nuestros propios resultados son consistentes con los reportados por el INEI, quien señala que tales brechas socioeconómicas aún no han sido superadas, tanto en el acceso a la educación como en el logro educativo (INEI, 2017).

Aunque las políticas del Proyecto Educativo Nacional Peruano (Consejo Nacional de Educación, 2007) atienden expresamente a ampliar el acceso y priorizar a las provincias más pobres (ubicadas usualmente en zonas rurales), nuestros resultados alertan que la brecha socioeconómica sigue siendo un problema vigente en las ciudades (ubicadas en provincias menos pobres). Más aún, la desigualdad del acceso viene acompañada de una desigualdad en la calidad educativa, pues los más pobres asisten a escuelas con menores recursos y docentes menos preparados (Cueto, et al., 2016). Eso dificulta aún más el pronóstico de logro educativo entre aquellos que tienen menos recursos económicos, especialmente dentro de la educación superior, la cual exige más preparación de lo que estas escuelas pueden brindar actualmente. 
Para reducir la brecha socioeconómica en educación, se requiere del Estado peruano una mayor inversión y estrategia acotada que ataquen directamente el factor pobreza. Sin embargo, la inversión en la educación disminuyó en -1,4\% entre el 2005 y 2014, manteniéndose baja en comparación con países latinoamericanos vecinos como Brasil, Argentina, Chile o Colombia (Valdez, 2014), aun cuando estos países experimentaron similar o menor crecimiento económico que Perú durante el mismo período (Banco Mundial, 2018). Por otro lado, la estrategia peruana adquiere forma de programas que atacan la brecha socioeconómica desde el acceso a la educación (v.g. el Programa No Escolarizado de Educación Inicial - PRONOEI) y/o brinda soporte a necesidades básicas para aumentar la probabilidad de logro educativo, especialmente en los primeros años de vida (v.g. Qali Warma para la alimentación escolar) (Asociación Kallpa, 2016 y Ministerio de Desarrollo e Inclusión Social, 2018).

Sin embargo, sus fondos son limitados y se deben destinar prioritariamente a las zonas rurales y provincias más pobres, descuidando la brecha que, como hemos detectado en nuestro estudio, aún sigue presente en las grandes ciudades. Respecto a la educación superior, programas como Beca 18 hacen posible que los talentos con escasos recursos provenientes de todos los rincones del país puedan capacitarse en Lima, para retornar a sus regiones posteriormente (República del Perú, 2011). Aunque muy valioso, este programa solo atiende las necesidades de un grupo específico y todavía muy pequeño, por lo que la brecha educativa en los niveles técnico y superior sigue siendo masiva en la medida en que se es más pobre.

\section{Brechas de género y socioeconómicas en la educación}

La brecha educativa de género entre adultos sigue existiendo, favoreciendo al sexo masculino, especialmente entre quienes tienen menos recursos económicos. En forma particular, nuestros resultados destacan estas diferencias en el nivel secundario, donde mujeres del SES bajo tienen notoria desventaja de logro frente a sus pares hombres. Esta desventaja en el logro parece estar más ligada al acceso y soporte, pues históricamente los desempeños escolares son similares: mujeres mejores en comprensión lectora, hombres mejores en matemáticas, pero con diferencias $\leq 5 \%$ (Guadalupe et al., 2017). El problema del acceso ya ha sido reportado por el INEI, quienes señalan que un 72,9\% de mujeres pertenecientes al SES bajo acceden a la educación secundaria, mostrando una brecha del -3,1\% respecto a los hombres (INEI, 2017).

Por otro lado, el escaso soporte económico que, por ejemplo, promueve una mayor asistencia a la escuela secundaria, prioriza a los hombres sobre las mujeres en el quintil socioeconómico más bajo (INEI, 2017). De hecho, nuestro estudio ha mostrado mayor logro 
educativo de las mujeres con SES alto versus aquellas con SES bajo (secundaria y superior), mientras que esta misma brecha es menor en los hombres (superior y especialmente en secundaria). Lo anterior implica también que, dado un SES bajo, los hombres obtienen un mayor logro educativo; lo que a su vez sugiere que la falta de soporte es también culturalfamiliar, a favor de los hombres (v.g. el apoyo antes, durante y después del embarazo adolescente) (Galvéz, 2014; Mendoza y Subiría, 2013; Monrroy, 2019).

En otro orden, los esfuerzos del Estado peruano para reducir esta brecha de género parecen ir en la dirección correcta, aunque en una dimensión todavía modesta. En lo cultural, el MINEDU ya ha reconocido que los estereotipos que favorecen la autoimagen de los hombres frente a las mujeres (Cueto, et al., 2016) aún están presentes en las escuelas (v.g. en los textos escolares) y deben ser trabajados (Ministerio de Educación, 2017), especialmente en el cómo los docentes podrían ver influenciada su práctica pedagógica con base en una sesgada expectativa de logro de sus estudiantes mujeres (Ministerio de Educación, 2016; ZapataCardona \& Rocha-Salamanca, 2014). Sin embargo, los esfuerzos ejecutivos para atacar estas brechas son todavía puntuales y escasos.

Para el soporte de tales esfuerzos, se cuenta con un marco político, normativo y de gestión en los compromisos internacionales asumidos, la Constitución Política del Perú creada en 1993), la Ley General de Educación (Ley 28044) creada en el 2003), el Proyecto Educativo Nacional al 2021 (Consejo Nacional de Educación, 2007), la Ley de Igualdad de Oportunidades entre Mujeres y Hombres (Ley 28983 de 2007), el Plan de Nacional de Igualdad de Oportunidades entre Mujeres y Varones 2006-2010 (Ministerio de la Mujer y Desarrollo Social, 2006), el Plan Nacional de Igualdad de Género Planig 2012-2017 (Centro de Derechos y Desarrollo, 2017), y la Ley de Fomento de la Educación de las Niñas y Adolescentes Rurales 2001 (Ley 27558, 2001).

No obstante, este trabajo coincide con Cueto, et al. (2016) al señalar que, a pesar de este marco normativo-legal, las acciones concretas aún son limitadas y no están suficientemente alineadas con los indicadores clave para la gestión (v.g. los aquí presentados de SES vs sexo). Esta limitación recrudece ante la disminución de fondos para la educación que ya señalamos en líneas anteriores (Valdez, 2014), afectando el alcance que toda iniciativa ejecutiva en favor de la equidad pueda tentar.

\section{Brechas generacionales y socioeconómicas en la educación}

Las brechas generacionales por nivel socioeconómico en la educación siguen existiendo, y favorece a quienes tienen más recursos económicos. En nuestro estudio, el aspecto 
positivo de esta brecha es que los jóvenes con SES alto y bajo muestran un mayor logro educativo de nivel superior, cuando se los compara con los más adultos de su mismo SES. El aspecto negativo es que entre los más jóvenes con diferente SES hay una brecha de $+12 \%$, favoreciendo a quienes tienen más recursos económicos. Respecto al acceso, los resultados nacionales reportados por el INEI son consistentes con nuestro hallazgo sobre el logro: la generación de 25 a 29 años con SES alto tuvo acceso a la educación superior universitaria en un 15,1\%, mientras que los de SES bajo lo tuvieron en un 3,3\% (INEI, 2018). Así, aunque nuestras generaciones más jóvenes tienen más acceso y logro a nivel de la educación superior, la inequidad asociada a la pobreza sigue estando presente.

La brecha generacional positiva es producto del aumento de la oferta educativa superior y el crecimiento económico, pero su brecha socioeconómica negativa aún requiere de una mayor intervención del Estado. En los últimos veinte años, la oferta educativa superior ha venido en aumento, especialmente la privada en provincias (INEI, 2018); mientras que, en el mismo período, el crecimiento económico (Banco Mundial, 2018) ha permitido que los estudiantes (o sus apoderados) puedan asumir el pago de sus estudios superiores. Obviamente, aquellas familias que no se beneficiaron del crecimiento económico han requerido -y aun requieren- de un apoyo especial por parte del Estado. El Programa Nacional de Becas y Crédito Educativo (PRONABEC) ha venido desplegando esfuerzos para cubrir esta brecha con: a) la Beca 18, que entre 2011 y 2015 financió los estudios de pregrado de casi 45 mil estudiantes de bajos recursos económicos provenientes de todas las regiones del país; b) la Beca Presidente de la República, que hasta el 2015 había financiado a 1216 becarios para maestrías y doctorados en el exterior (República del Perú, 2016).

A pesar de lo valioso de tales esfuerzos, la inversión en estos programas aún es modesta en comparación con países vecinos como Chile (OCDE, 2011). Potenciar los programas del PRONABEC que atacan directamente la brecha socioeconómica en la educación superior, junto a otros que lo hacen indirectamente como los del CONCYTEC (Consejo Nacional de Ciencia, Tecnología e Innovación (CONCYTEC), 2018), ayudará a reducir esta brecha y equiparar nuestro capital humano con nuestros vecinos de la región.

Nuestro estudio tiene fortalezas y limitaciones que creemos oportuno declarar. El tamaño muestral grande y la representatividad de una ciudad que a su vez se asemeja a muchas otras ciudades de la costa norte del Perú, son una fortaleza que favorece la precisión del dato y su generalización hacia zonas urbanas. Asimismo, nuestro estudio ofrece indicadores de logro educativo por niveles (en vez de por años de estudio) en adultos, estratificados por SES*sexo o SES*edad, información que es muy escasa en la literatura actual y que, como hemos mostrado, 
es relevante para la gestión educativa. Por otro lado, nuestra principal limitación del estudio es generar la variable SES a través de una variable proxy, lo cual pudo haber generado un sesgo de sensibilidad en la medición reduciendo la consistencia de los resultados obtenidos, sin embargo se ha mostrado consistente en comparación con otros estudios nacionales en los que se emplearon mediciones estándar.

Nuestra recomendación hacia el Estado y las organizaciones interesadas se resume en afinar los indicadores educativos, potenciar los programas vigentes, incrementar la inversión y no descuidar a las zonas urbanas. Los indicadores que guían la gestión y permiten evaluar el impacto de los programas del Estado pueden alinearse aún más para combatir la inequidad. Una forma de hacerlo es incluir los que aquí hemos mostrado, o una forma mejorada de ellos, de forma tal que el monitoreo de las brechas sea sencillo y permanente. Como hemos mostrado, programas como los del PRONABEC atacan la brecha socioeconómica, pero aún requieren mayor inversión que garantice mayor cobertura. Los indicadores mejorados permitirán evaluar el impacto que tal inversión tendrá en la reducción de brechas. De hecho, la inversión para reducir las brechas no debe ser exclusiva del MINEDU y debe facilitar el logro en todos los niveles educativos, lo que significa una visión holística y mayor compromiso por parte del Estado. En esa visión, un reconocimiento de que las brechas educativas no sólo están entre el campo y la ciudad, sino también dentro de las mismas ciudades, es necesario para promover homogéneamente todo esfuerzo en favor de la equidad.

En conclusión, las brechas educativas entre adultos de zonas urbanas siguen desfavoreciendo a las mujeres y a los más pobres. Los adultos con nivel socioeconómico alto tienen un mayor nivel de logro educativo, especialmente de secundaria y superior. Entre los más pobres, las mujeres logran menor nivel educativo que los hombres; mientras que, entre los más ricos, las mujeres logran un nivel educativo similar al de los hombres. Las generaciones más jóvenes están logrando un mayor desarrollo educativo, pero con ventajas para aquellos que tienen un mayor nivel socioeconómico. Estas desigualdades deben atacarse con más inversión dirigida a potenciar los programas vigentes, dentro de una gestión educativa que actualice sus indicadores y no descuide a las zonas urbanas, facilitando la evaluación del impacto que estas acciones tienen en la reducción de las inequidades.

\section{Referencias}

Angrisani, M., Lee, J., \& Meijer, E. (2020). The gender gap in education and late-life cognition: Evidence from multiple countries and birth cohorts. The Journal of the Economics of Ageing, 16, 100232. DOI: https://doi.org/10.1016/j.jeoa.2019.100232 
Asociación Kallpa. (2016). Programas No Escolarizados Educación Inicial (PRONOEI). Disponible en: http://www.kallpa.org.pe/webnueva/que-hacemos/escuelassaludables/programas-no-escolarizados-educacion-inicial-pronoei/

Akabayashi, H., Nozaki, K., Yukawa, S., \& Li, W. (2020). Gender differences in educational outcomes and the effect of family background: A comparative perspective from East Asia. Chinese Journal of Sociology, 6(2), 315-335. DOI: https://doi.org/10.1177/2057150X20912581

Banco Mundial. (2018). Perú Panorama general. Disponible en: http://www.bancomundial.org/es/country/peru/overview

Benavides, M. (2007). Lejos (aún) de la equidad: La persistencia de las desigualdades educativas en el Perú. In Investigación, políticas y desarrollo en el Perú. (pp. 457-483). Lima: GRADE. Disponible en: http://www.grade.org.pe/upload/publicaciones/archivo/download/pubs/InvPolitDesarr11.pdf

Bodovski, K., Munoz, I., Byun, S. Y., \& Chykina, V. (2020). Do Education System Characteristics Moderate the Socioeconomic, Gender and Immigrant Gaps in Math and Science Achievement?. International Journal of Sociology of Education, 9(2), 122-154. DOI: $\underline{10.17583 / \text { rise.2020.4807 }}$

Centro de derechos y desarrollo (2017). Plan Nacional de Igualdad de Género: Caminando hacia la igualdad de género. Lima, Perú: Centro de derechos y desarrollo. Disponible en: https://www.mimp.gob.pe/files/direcciones/dgignd/publicaciones/Caminado-haciala-igualdad-de-genero.pdf

CEPAL - Comisión Económica para América Latina y el Caribe (2017). Planes de igualdad de género en América Latina y el Caribe: Mapas de ruta para el desarrollo. Naciones Unidas: Comisión Económica para América Latina y el Caribe. [En línea]. Disponible en: https://www.cepal.org/sites/default/files/events/files/planes_de_igualdad_de_genero_en_ america_latina_y_el_caribe._mapas_de_ruta_para_el_desarrollo.pdf

CEDAL - Centro de derechos y desarrollo (2017). Plan Nacional de Igualdad de Género: Caminando hacia la igualdad de género. Lima, Perú: Centro de derechos y desarrollo. Disponible en: https://www.mimp.gob.pe/files/direcciones/dgignd/publicaciones/Caminado-hacia-laigualdad-de-genero.pdf

Congreso de la República del Perú. (2007, 16 de marzo). Ley N²8983. Ha dado la siguiente ley de igualdad de oportunidades entre mujeres y hombres. Diario oficial: El Peruano. Disponible en: https://leyes.congreso.gob.pe/Documentos/Leyes/28983.pdf

Congreso de la República del Perú (2001, 23 de noviembre). Ley $N^{\circ} 27558$. Ha dado la siguiente ley de Fomento de la Educación de las Niñas y Adolescentes Rurales. Disponible en: https://www.gob.pe/institucion/minedu/normas-legales/118371-27558

Consejo Nacional de Educación (2007). Proyecto Educativo Nacional al 2021: La educación que queremos para el Perú. Lima: Consejo Nacional de Educación. Disponible en: 
https://www.cne.gob.pe/uploads/proyecto-educativo-nacional/version-pen/penoficial.pdf

CONCYTEC - Consejo Nacional de Ciencia, Tecnología e Innovación (2018). Trámites y servicios. Ministerio de Educación. Disponible en: https://portal.concytec.gob.pe/

Cueto, S., Miranda, A., \& Vásquez, M. C. (2016). Inequidades en educación. En GRADE. Investigación para el desarrollo en el Perú: once balances. Lima: GRADE. Disponible en: http://www.grade.org.pe/wp-content/uploads/inequidadesedu_SC_35.pdf

Díaz, J. J. (2008). Educación superior en el Perú: tendencias de la demanda y la oferta. In Análisis de programas, procesos y resultados educativos en el Perú: contribuciones empíricas para el debate. (pp. 83-129). Lima: GRADE. Disponible en: https://www.grade.org.pe/wp-content/uploads/analisis-2.pdf

Domínguez, J., Azañedo, D., Bazalar-Palacios, J., y Rodríguez, Y. (2019). Enfermedades no transmisibles en la ciudad de Chimbote: prevalencia y factores bio-sociodemográficos. Chimbote: Universidad Católica los Ángeles de Chimbote. Disponible en: http://repositorio.uladech.edu.pe/bitstream/handle/123456789/11338/ENFERMEDADES \%20NO\%20TRANSMISIBLES\%20EN\%20LA\%20CIUDAD\%20DE\%20CHIMBOTE \%20-\%20Julio\%20Dom\%c3\%adnguez\%20Granda\%20\%20Digital.pdf? sequence $=3 \&$ isAllowed $=y$

Galvéz, D. (2014). EDUDATOS $N^{\circ} 20:$ La deserción escolar en las adolescentes embarazadas del Perú. Lima: Ministerio de Educación del Perú. Disponible en: http://escale.minedu.gob.pe/c/document_library/get_file?uuid=cec124e6-2679-4b52$\underline{957 a-37 c 9 c 62604 d a \& g r o u p I d=10156}$

Guadalupe, C., León, J., Rodríguez, J. S., y Vargas, S. (2017). Estado de la Educación en el Perú: Análisis y perspectivas de la educación básica. Lima: FORGE. Disponible en: http://www.grade.org.pe/forge/descargas/Estado\%20de\%20la\%20educaci\%C3\%B3n\%2 0en\%20el\%20Per\%C3\%BA.pdf

INEI - Instituto Nacional de Estadística e Informática (2017). Brechas de Género 2017: Avances hacia la igualdad de mujeres y hombres. Lima, Perú: Instituto Nacional de Estadística e Informática. [En línea]. Disponible en: https://www.inei.gob.pe/media/MenuRecursivo/publicaciones_digitales/Est/Lib1444/libr o.pdf

INEI - Instituto Nacional de Estadística e Informática (2018). Perú: Indicadores de Educación por departamento, 2007-2017. Lima, Perú: Instituto Nacional de Estadística e Informática. [En línea]. Disponible en: https://www.inei.gob.pe/media/MenuRecursivo/publicaciones_digitales/Est/Lib1529/libr o.pdf

INEI - Instituto Nacional de Estadistica e Informatica (2018). Ancash: Resultados definitivos. Lima: Instituto Nacional de Estadistica e Informatica. [En línea]. Disponible en: https://www.inei.gob.pe/media/MenuRecursivo/publicaciones_digitales/Est/Lib1552/02 TOMO_01.pdf 
INEI - Instituto Nacional de Estadística e Informática (2020). Perú: Indicadores de Educación por Departamentos, 2009-2019. Lima, Perú: Instituto Nacional de Estadistica e Informatica. [En línea]. Disponible en:

https://www.inei.gob.pe/media/MenuRecursivo/publicaciones_digitales/Est/Lib1751/libr o.pdf

Lundberg, S. (2020). Educational gender gaps. Southern Economic Journal, 87(2), 416-439. DOI: https://doi.org/10.1002/soej.12460

Mendoza, W., y Subiría, G. (2013). El embarazo adolescente en el Perú: Situación actual e implicancias para las políticas públicas. Revista Peruana de Medicina Experimental y Salud Publica, 30(3), 471-479. DOI: https://doi.org/10.1080/17441692.2011.590813

Ministerio de Desarrollo e Inclusión Social (2018). Programa Nacional de Alimentación Escolar (Qali Warma). Disponible en: https://www.qaliwarma.gob.pe/

Ministerio de Educación (2016). ¿Cuánto aprenden nuestros estudiantes al término de la Educación Primaria?: Informe de logros de aprendizaje y sus factores asociados en la Evaluación Muestral 2013. Lima, Perú. Disponible en: http://umc.minedu.gob.pe/wpcontent/uploads/2016/07/Informe-de-resultados-EM-2013-1.pdf

Ministerio de Educación. (2017). Género y brechas de aprendizaje en matemática al término de la educación primaria. Lima, Perú. Disponible en: http://umc.minedu.gob.pe/generoy-brechas-de-aprendizaje-en-matematica-al-termino-de-la-educacion-primaria-2/

Ministerio de la Mujer y Desarrollo Social. (2006). Plan Nacional de Igualdad de Oportunidades entre mujeres y Varones 2006 - 2010. Lima, Perú: Ministerio de la Mujer y Desarrollo Social. Disponible en: https://www.mimp.gob.pe/files/direcciones/dgignd/planes/pio2006_2010_dcto_completo f.pdf

Monrroy, N.M. (2019). Maternidad adolescente y abandono escolar: una aproximación desde la subjetividad, el género y el curso de vida de un grupo de mujeres en Belén, Iquitos. Bulletin de l'Institut français d'études andines, (48 (3)), 303-321. DOI: https://doi.org/10.4000/bifea.10933

OCDE - Organización para ña Cooperación y el Desarrollo Económicos (2016). Avanzando hacia una mejor educación para Perú. OCDE. Disponible en: https://www.oecd.org/dev/Avanzando-hacia-una-mejor-educacion-en-Peru.pdf

OCDE - Organización para la cooperación y el desarrollo Económicos. (2011). Revisión de Políticas Nacionales de Educación: Programa Becas Chile. Disponible en: http://www.oecd.org/innovation/research/47309025.pdf

República del Perú. (2016). Informe Preelectoral Administración 2011-2016. Disponible en: https://www.mef.gob.pe/contenidos/pol_econ/documentos/Informe_PreElectoral_2011_ 2016.pdf

Romero-Martínez, A. M., Montoro-Sánchez, Á., \& Garavito-Hernández, Y. (2017). El efecto de la diversidad de género y el nivel educativo en la innovación. Revista de Administração de Empresas, 57(2), 123-134. DOI: http://dx.doi.org/10.1590/s0034- 


\section{$\underline{759020170202}$}

StataCorp. (2017). Stata Statistical Software: Release 15. College Station. Retrieved January 3, 2019, from TX: StataCorp LLC

Steele, L., Palmer, J., Lloyd, A., Fotheringham, J., Iqbal, J., \& Grech, E. D. (2018). Impact of socioeconomic status on survival following ST-elevation myocardial infarction in a universal healthcare system. International Journal of Cardiology. DOI: 10.1016/j.ijcard.2018.11.111

UNESCO - United Nations Educational Scientific and Cultural Organization (2019). Informe de seguimiento de la educación en el mundo 2019: informe sobre género: construyendo puentes para la igualdad de género. Paris: United Nations Educational, Scientific and Cultural Organization. [En línea]. Disponible en: https://unesdoc.unesco.org/ark:/48223/pf0000369621

Vaezghasemi, M., Mosquera, P. A., Gustafsson, P. E., Nilsson, K., \& Strandh, M. (2020). Decomposition of income-related inequality in upper secondary school completion in Sweden by mental health, family conditions and contextual characteristics. SSMpopulation health, 11, 100566. DOI: https://doi.org/10.1016/j.ssmph.2020.100566

Valdez, F. (2014). EDUDATOS N¹8: El PBI y Gasto Público en la Educación Pública. Una mirada a la región. Lima, Perú: Ministerio de Educación. Disponible en: http://escale.minedu.gob.pe/c/document_library/get_file?uuid=c2014d65-fe6d-43fbbee6-72fd76f46806\&groupId=10156

World Economic Forum (2016). The Global Gender Gap Report 2016. Switzerland: World Economic Forum. [En línea]. Disponible en:

http://www3.weforum.org/docs/GGGR16/WEF_Global_Gender_Gap_Report_2016.pdf

Zapata-Cardona, L., \& Rocha-Salamanca, P. (2014). Equidad de género en la clase de Matemáticas-Gender equity in the mathematics classroom. Revista científica, 2(19), 168178. DOI: $\underline{\text { https://doi.org/10.14483/23448350.6503 }}$ 